\title{
BASIC POLYHEDRAL THEORY
}

\author{
VOLKER KAIBEL
}

A polyhedron is the intersection of finitely many affine halfspaces, where an affine halfspace is a set

$$
\mathrm{H}^{\leq}(a, \beta)=\left\{x \in \mathbb{R}^{n}:\langle a, x\rangle \leq \beta\right\}
$$

for some $a \in \mathbb{R}^{n}$ and $\beta \in \mathbb{R}$ (here, $\langle a, x\rangle=\sum_{j=1}^{n} a_{j} x_{j}$ denotes the standard scalar product on $\mathbb{R}^{n}$ ). Thus, every polyhedron is the set

$$
\mathrm{P}^{\leq}(A, b)=\left\{x \in \mathbb{R}^{n}: A x \leq b\right\}
$$

of feasible solutions to a system $A x \leq b$ of linear inequalities for some matrix $A \in \mathbb{R}^{m \times n}$ and some vector $b \in \mathbb{R}^{m}$. Clearly, all sets $\left\{x \in \mathbb{R}^{n}: A x \leq b, A^{\prime} x=b^{\prime}\right\}$ are polyhedra as well, as the system $A^{\prime} x=b^{\prime}$ of linear equations is equivalent the system $A^{\prime} x \leq b^{\prime},-A^{\prime} x \leq-b^{\prime}$ of linear inequalities. A bounded polyhedron is called a polytope (where bounded means that there is a bound which no coordinate of any point in the polyhedron exceeds in absolute value).

Polyhedra are of great importance for Operations Research, because they are not only the sets of feasible solutions to Linear Programs (LP), for which we have beautiful duality results and both practically and theoretically efficient algorithms, but even the solution of (Mixed) Integer Linear Programming (MILP) problems can be reduced to linear optimization problems over polyhedra. This relationship to a large extent forms the backbone of the extremely successful story of (Mixed) Integer Linear Programming and Combinatorial Optimization over the last few decades.

In Section 1, we review those parts of the general theory of polyhedra that are most important with respect to optimization questions, while in Section 2 we treat concepts that are particularly relevant for Integer Programming. Most of the "basic polyhedral theory" today is standard textbook knowledge. In Section 3, for some of the results we provide references to the original papers. There, we also give pointers to proofs of the theorems mentioned in the first two sections, where we mainly refer to the beautiful book by Schrijver [33]. There are, of course, many other excellent treatments of the theory of polyhedra with respect to optimization questions, e.g., in the recent survey by Conforti, Cornuéjols, and Zambelli [7], in the handbook articles by Schrijver [34] and Burkard [4], as well as in the books by Nemhauser and Wolsey [30], Grötschel, Lovàsz, and Schrijver [20], Bertsimas and Weismantel [3], Cook, Cuningham, Pulleyblank, and Schrijver [9], Wolsey [40], Korte and Vygen [26], or Barvinok [1]. The books by Ziegler [41] and Grünbaum [21] are most important sources for the general geometric and combinatorial theory of polyhedra, in particular of polytopes. We also refer to the handbook article by Gritzmann and Klee [19] as well as the one by Bayer and Lee [2].

\section{The GeOMETRY OF POLYHEDRA}

1.1. Some Notation. We define $[p]=\{1, \ldots, p\}$ and denote by $\mathbb{R}_{+}=\{\alpha \in \mathbb{R}: \alpha \geq 0\}$ the set of nonnegative real numbers. A submatrix of $M \in \mathbb{R}^{m \times n}$ is a matrix $M_{I, J} \in \mathbb{R}^{I \times J}$ for some $\varnothing \neq I \subseteq[m]$ and $\varnothing \neq J \subseteq[n]$ formed by the rows and columns of $M$ indexed by the elements of $I$ and $J$, respectively. In particular, $M_{i, j} \in \mathbb{R}$ is the entry in row $i$ and column $j$. We write

Date: January 13, 2010.

Article prepared for Wiley Encyclopedia of Operations Research and Management Science. 
$M_{I, \star}=M_{I,[n]}$ and $M_{\star, J}=M_{[m], J}$, in particular, $M_{i, \star} \in \mathbb{R}^{n}$ and $M_{\star, j} \in \mathbb{R}^{m}$ are the $i$-th row and the $j$-th column of $M$, respectively. The kernel of $M \in \mathbb{R}^{m \times n}$ is $\operatorname{ker}((M))=\left\{x \in \mathbb{R}^{n}: M x=\mathbb{O}\right\}$. The identity matrix $\operatorname{Id}_{n} \in \mathbb{R}^{n \times n}$ has one-entries on its main diagonal and zeroes elsewhere.

For $x \in \mathbb{R}^{n}$ and $J \subseteq[n]$, the vector formed by the components of $x$ indexed by elements of $J$ is denoted by $x_{J} \in \mathbb{R}^{J}$. We denote by $\langle x, y\rangle=\sum_{j=1}^{n} x_{j} y_{j}$ the standard scalar product of $x, y \in \mathbb{R}^{n}$. We consider, in the context of matrix multiplication, all vectors as column vectors, and use $(\ldots)^{\mathrm{t}}$ to refer to the transposed matrix or vector. We denote by $\mathbb{O} \in \mathbb{R}^{n}$ the zero vector in $\mathbb{R}^{n}$ and by $\mathbb{e}_{i} \in \mathbb{R}^{n}$ the standard unit vector having its $i$-th component equal to one, all other components being zero.

Denoting by $\delta(M)$ the set of all determinants of submatrices (formed by arbitrary subsets of rows and columns of equal cardinality, including the empty submatrix, whose determinant is considered to be one) of a matrix $M \in \mathbb{R}^{m \times n}$, we define

$$
\Delta(M)=\left\{\frac{p}{q}: p, q \in \delta(M) \cup(-\delta(M)), q \neq 0\right\},
$$

and, for every finite set $\varnothing \neq V \subseteq \mathbb{R}^{n}$, we set $\Delta(V)=\Delta(M)$, where $M$ is any matrix whose set of columns is $V$. Clearly, for rational matrices $M \in \mathbb{Q}^{m \times n}$ and (finite) sets $V \subseteq \mathbb{Q}^{n}$ we have $\Delta(M), \Delta(V) \subseteq \mathbb{Q}$.

The encoding length of $\alpha=\frac{p}{q} \in \mathbb{Q}$ with $p, q \in \mathbb{Z}$ relatively prime is

$$
\langle\alpha\rangle=1+\left\lceil\log _{2}(|p|+1)\right\rceil+\left\lceil\log _{2}(|q|+1)\right\rceil .
$$

For a rational vector $v \in \mathbb{Q}^{n}$ and a rational matrix $M \in \mathbb{Q}^{m \times n}$, we define

$$
\langle v\rangle=n+\sum_{j=1}^{n}\left\langle v_{j}\right\rangle \quad \text { and } \quad\langle M\rangle=m n+\sum_{i=1}^{m} \sum_{j=1}^{n}\left\langle M_{i, j}\right\rangle .
$$

Moreover, we denote by $\langle M\rangle_{\max }$ the maximum encoding length of any entry in $M$, as well as by $\langle V\rangle_{\max }$ the maximum encoding length of all components of vectors in the finite set $V \subseteq \mathbb{Q}^{n}$.

1.2. Basics. Most important, every polyhedron $P \subseteq \mathbb{R}^{n}$ is convex, i.e., for all $x, y \in P$ and $\alpha \in$ $[0,1]$, we have $\alpha x+(1-\alpha) y \in P$ as well. Moreover, polyhedra are topologically closed subsets of $\mathbb{R}^{n}$.

As the solution sets to finite systems of linear inequalities, polyhedra generalize affine subspaces, which are the solution sets to systems of linear equations. The criterion for $A x=b$ not being solvable via the existence of some multiplier vector $\lambda \in \mathbb{R}^{m}$ with $\lambda^{\mathrm{t}} A=\mathbb{O}$ and $\langle\lambda, b\rangle \neq 0$ generalizes to systems of linear inequalities in the following way (where both parts of the theorem follow easily from each other).

Theorem 1 (Farkas-Lemma). For each $A \in \mathbb{R}^{m \times n}$ and $b \in \mathbb{R}^{m}$ the following hold:

(i) Either $A x \leq b$ is solvable or there is some $\lambda \in \mathbb{R}_{+}^{m}$ with $\lambda^{\mathrm{t}} A=\mathbb{O}$ and $\langle\lambda, b\rangle<0$ (but not both).

(ii) Either $A x=b, x \geq \mathbb{O}$ is solvable or there is some $\lambda \in \mathbb{R}^{m}$ with $\lambda^{\mathrm{t}} A \geq \mathbb{O}$ and $\langle\lambda, b\rangle<0$ (but not both).

It turns out that the "crucial solutions" to systems of linear inequalities are obtained as the unique solutions of certain regular linear equation systems, whose components are well-known to be expressible in the following way. 
Theorem 2 (Cramer's rule). If $A \in \mathbb{R}^{n \times n}$ is regular, then, for every $b \in \mathbb{R}^{n}$, the unique solution $x \in \mathbb{R}^{n}$ with $A x=b$ is given by

$$
x_{j}=\frac{\operatorname{det}\left(A_{\star, 1}, \ldots, A_{\star, j-1}, b, A_{\star, j+1}, \ldots, A_{\star, n}\right)}{\operatorname{det}(A)} \quad \text { for all } j \in[n] .
$$

The following estimates follows from the Leibniz formula for determinants.

Theorem 3. There is a constant $C>0$ such that the estimates

$$
\langle\alpha\rangle \leq C \cdot n^{2} \cdot\langle M\rangle_{\max } \text { for all } \alpha \in \Delta(M)
$$

and

$$
\langle\beta\rangle \leq C \cdot n^{2} \cdot\langle V\rangle_{\max } \quad \text { for all } \beta \in \Delta(V)
$$

hold for all $M \in \mathbb{Q}^{m \times n}$ and for all finite sets $V \subseteq \mathbb{Q}^{n}$.

1.3. Polyhedral and Finitely Generated Cones. A cone is a subset $K \subseteq \mathbb{R}^{n}$ with $\mathbb{O} \in K$ and $\alpha y \in K$ for all $y \in K$ and $\alpha \in \mathbb{R}_{+}$. A polyhedral cone is a polyhedron that is a cone, or, equivalently, a polyhedron $\mathrm{P} \leq(A, \mathbb{O})$ for some $A \in \mathbb{R}^{m \times n}$.

The (convex) conic hull of a subset $X \subseteq \mathbb{R}^{n}$ is the cone

$$
\operatorname{ccone}(X)=\left\{\sum_{x \in X^{\prime}} \alpha_{x} x: X^{\prime} \subseteq X,\left|X^{\prime}\right|<\infty, \alpha_{x} \geq 0 \text { for all } x \in X^{\prime}\right\}
$$

(with ccone $(\varnothing)=\{\mathbb{O}\}$ ) of all conic combinations of the vectors in $X$. A cone $K \subseteq \mathbb{R}^{n}$ is finitely generated, if there is a finite set $X \subseteq \mathbb{R}^{n}$ with $K=\operatorname{ccone}(X)$. Every vector in a conic hull can be obtained by a conic combination of few generators:

Theorem 4 (Carathéodory's Theorem, conic version). For each $X \subseteq \mathbb{R}^{n}$ and $y \in \operatorname{ccone}(X)$ there is a linearly independent subset $X^{\prime} \subset X$ (in particular: $\left|X^{\prime}\right| \leq n$ ) with $y \in \operatorname{ccone}\left(X^{\prime}\right)$.

The Farkas-Lemma (Part (ii) of Theorem 1) yields a separation theorem for finitely generated cones.

Theorem 5. If $y \notin$ ccone $(X)$ for the finite set $X \subseteq \mathbb{R}^{n}$, then there is some $a \in \mathbb{R}^{n}$ with

$$
\langle a, x\rangle \leq 0<\langle a, y\rangle \text { for all } x \in \operatorname{ccone}(X)
$$

(i.e., ccone $(X) \subseteq \mathrm{H}^{\leq}(a, 0)$, but $y \notin \mathrm{H}^{\leq}(a, 0)$ ).

The following result implies that every polyhedral cone is finitely generated, which is of utmost importance for the theory of polyhedra.

Theorem 6. For every matrix $A \in \mathbb{R}^{m \times n}$, there is a finite set $X \subseteq(\Delta(A))^{n}$ with

$$
\mathrm{P} \leq(A, \mathbb{O})=\operatorname{ccone}(X) .
$$

The polar of a cone $K \subseteq \mathbb{R}^{n}$ is the convex cone

$$
K^{\circ}=\left\{a \in \mathbb{R}^{n}:\langle a, x\rangle \leq 0 \text { for all } x \in K\right\} .
$$

The polar of a finitely generated cone ccone $(X)$ with a finite set $X \subseteq \mathbb{R}^{n}$ obviously is the polyhedral cone

$$
(\operatorname{ccone}(X))^{\circ}=\left\{a \in \mathbb{R}^{n}:\langle x, a\rangle \leq 0 \text { for all } x \in X\right\} .
$$

From Theorem 5 we also obtain

$$
(\mathrm{P} \leq(A, \mathbb{O}))^{\circ}=\operatorname{ccone}\left\{A_{1, \star}, \ldots, A_{m, \star}\right\}
$$

for each $A \in \mathbb{R}^{m \times n}$.

Moreover, from Theorem 5 one deduces $(\operatorname{ccone}(X))^{\circ \circ}=\operatorname{ccone}(X)$, from which one finds, by applying Theorem 6 as well as (two times) (1), the following reverse statement to Theorem 6 . 
Theorem 7. For every finite set $X \in \mathbb{R}^{n}$, there is a matrix $A \in(\Delta(X))^{m \times n}$ with

$$
\operatorname{ccone}(X)=\mathrm{P}^{\leq}(A, \mathbb{O}) \text {. }
$$

1.4. The Fundamental Structure of Polyhedra. The homogenization of a polyhedron $\mathrm{P} \leq(A, b) \subseteq$ $\mathbb{R}^{n}$ (with $A \in \mathbb{R}^{m \times n}, b \in \mathbb{R}^{m}$ ) is the polyhedral cone

$$
\operatorname{homog}(\mathrm{P} \leq(A, b))=\left\{(x, \xi) \in \mathbb{R}^{n} \oplus \mathbb{R}: A x-\xi b \leq \mathbb{O}, \xi \geq 0\right\} .
$$

Applying Theorem 6 to $\operatorname{homog}\left(\mathrm{P}^{\leq}(A, b)\right)$ as well as Theorem 7 to the finitely generated cone

$$
\operatorname{ccone}\left(\left\{(x, 1) \in \mathbb{R}^{n} \oplus \mathbb{R}: x \in X\right\} \cup\left\{(y, 0) \in \mathbb{R}^{n} \oplus \mathbb{R}: y \in Y\right\}\right)
$$

for finite sets $X, Y \subseteq \mathbb{R}^{n}$, one obtains the following representation theorem for polyhedra, where

$$
\operatorname{conv}(X)=\left\{\sum_{x \in X^{\prime}} \alpha_{x} x: X^{\prime} \subseteq X,\left|X^{\prime}\right|<\infty, \sum_{x \in X^{\prime}} \alpha_{x}=1, \alpha_{x} \geq 0 \text { for all } x \in X^{\prime}\right\}
$$

denotes the convex hull of a set $X \subseteq \mathbb{R}^{n}$, and

$$
S+T=\{s+t: s \in S, t \in T\}
$$

is the Minkowski sum of $S, T \subseteq \mathbb{R}^{n}$.

Theorem 8 (Weyl-Minkowski Theorem).

(i) For every $A \in \mathbb{R}^{m \times n}$ and $b \in \mathbb{R}^{m}$ there are finite sets $X, Y \subseteq(\Delta(A, b))^{n}$ with

$$
\mathrm{P} \leq(A, b)=\operatorname{conv}(X)+\operatorname{ccone}(Y) .
$$

(ii) For all finite sets $X, Y \subseteq \mathbb{R}^{n}$ there are $A \in(\Delta(X \cup Y))^{m \times n}$ and $b \in(\Delta(X \cup Y))^{m}$ with

$$
\operatorname{conv}(X)+\operatorname{ccone}(Y)=\mathrm{P}^{\leq}(A, b) .
$$

Thus, every polyhedron can be represented by outer descriptions (intersection of finitely many affine halfspaces) and by inner descriptions (Minkowski sum of a polytope and a finitely generated cone). Clearly, algebraically an outer description may contain both linear inequalities and linear equations. Depending on the context, one type of description of a polyhedron can be significantly more convenient to deal with than the other. For instance, from inner descriptions one concludes readily that images of polyhedra under linear maps are polyhedra as well (see Section 1.6). In turn, the fact that also preimages of polyhedra under linear maps are polyhedra is easy to prove via outer descriptions. From outer descriptions of polyhedra one also finds immediately that intersections of finitely many polyhedra are polyhedra.

A rational polyhedron is a polyhedron for which $A$ and $b$, or, equivalently, $X$ and $Y$, in (3) and (4) can be chosen to be rational matrices, vectors, and sets, respectively. Denoting, for a rational polyhedron $P \subseteq \mathbb{R}^{n}$, by $\langle P\rangle_{\max }^{\text {outer }}$ the smallest number $\alpha$ such that there is an outer description $P=\mathrm{P} \leq(A, b)$ of $P$ with a rational matrix $A$ and a rational vector $b$ with $\langle(A, b)\rangle_{\max } \leq \alpha$, and by $\langle P\rangle_{\max }^{\text {inner }}$ the smallest number $\beta$ such that there is an inner description $P=\operatorname{conv}(X)+\operatorname{ccone}(Y)$ of $P$ with a rational finite sets $X, Y \subseteq \mathbb{Q}^{n}$ with $\langle X \cup Y\rangle_{\max } \leq \beta$, we obtain the following result from Theorem 3 .

Theorem 9. There is a constant $C>0$ such that

$$
\langle P\rangle_{\max }^{\text {inner }} \leq C \cdot n^{2} \cdot\langle P\rangle_{\max }^{\text {outer }} \quad \text { and }\langle P\rangle_{\max }^{\text {outer }} \leq C \cdot n^{2} \cdot\langle P\rangle_{\max }^{\text {inner }}
$$

holds for every rational polyhedron $P \subseteq \mathbb{R}^{n}$. 
In particular, whenever the rational system $A x \leq b$ (with $A \in \mathbb{Q}^{m \times n}$ and $b \in \mathbb{Q}^{m}$ ) has any solution, then it also has a solution whose encoding length is bounded by a polynomial in $\langle(A, b)\rangle$. This shows that the linear programming feasibility problem is contained in the complexity class NP, and, via Theorem 1, also in coNP. (Of course, it is well-known that this problem is even solvable in polynomial time).

However, the smallest possible number of inequalities $A x \leq b$ in an outer description and the smallest possible cardinalities of the sets $X$ and $Y$ in an inner description of a rational polyhedron $P$ are not bounded polynomially by each other, in general.

The characteristic cone (or recession cone) of a polyhedron $P \subseteq \mathbb{R}^{n}$ is

$$
\operatorname{char}(P)=\left\{y \in \mathbb{R}^{n}: x+\operatorname{cone}\{y\} \subseteq P \text { for all } x \in P\right\} .
$$

Theorem 10. If $\varnothing \neq P=\mathrm{P} \leq(A, b)=\operatorname{conv}(X)+\operatorname{ccone}(Y) \subseteq \mathbb{R}^{n}$ is a polyhedron (with $A \in$ $\mathbb{R}^{m \times n}, b \in \mathbb{R}^{m}$, and $X, Y \subseteq \mathbb{R}^{n}$ finite), then we have

$$
\operatorname{ccone}(Y)=\operatorname{char}(P)=\mathrm{P}^{\leq}(A, \mathbb{O}) .
$$

The lineality space of a polyhedron $P \subseteq \mathbb{R}^{n}$ is the largest linear subspace

$$
\text { lineal }(P)=\operatorname{char}(P) \cap(-\operatorname{char}(P))
$$

contained in $\operatorname{char}(P)$.

Theorem 11. For a polyhedron $\varnothing \neq P=\mathrm{P}^{\leq}(A, b)$ (with $A \in \mathbb{R}^{m \times n}, b \in \mathbb{R}^{m}$ ) we have

$$
\text { lineal }(P)=\operatorname{ker}(A) \text {. }
$$

A non-empty polyhedron $\varnothing \neq P \subseteq \mathbb{R}^{n}$ with lineality space lineal $(P)=\{\mathbb{O}\}$ is called pointed. For most purposes, it is sufficient to consider pointed polyhedra. In fact, if a polyhedron has a nontrivial lineality space $L \neq\{\mathbb{O}\}$ then, in most contexts, it is sufficient to investigate instead of $P$ its orthogonal projection to the orthogonal complement of $L$, which is a pointed polyhedron. Therefore, subsequently we will mainly consider pointed polyhedra. For instance, all polyhedra that are contained in the nonnegative orthant $\mathbb{R}_{+}^{n}$ as well as all polytopes are pointed.

1.5. Faces of Polyhedra. For $a \in \mathbb{R}^{n} \backslash\{\mathbb{O}\}$ and $\beta \in \mathbb{R}$, we denote by

$$
\mathrm{H}^{=}(a, \beta)=\left\{x \in \mathbb{R}^{n}:\langle a, x\rangle=\beta\right\}
$$

the boundary hyperplane of the affine halfspace $\mathrm{H} \leq(a, \beta)$. A (proper) face of a polyhedron $P \subseteq$ $\mathbb{R}^{n}$ is the intersection $F=P \cap \mathrm{H}^{=}(a, \beta)$ of $P$ with the boundary hyperplane of some halfspace $\mathrm{H}^{\leq}(a, \beta) \supseteq P$ containing $P$. The face $F$ is said to defined by the inequality $\langle a, x\rangle \leq \beta$ in this case. Additionally, $\varnothing$ and $P$ itself are considered (trivial) faces of $P$ (defined by $\langle\mathbb{O}, x\rangle \leq-1$ and $\langle\mathbb{O}, x\rangle \leq 0$, respectively). Non-empty faces are particularly important for optimization, because they are the (non-empty) sets of optimal solutions to linear optimization problems over polyhedra.

The following result (which is a generalization of Part (i) of Theorem 1) provides a characterization of those inequalities that are valid for (and thus define faces of) a non-empty polyhedron.

Theorem 12. Let $A \in \mathbb{R}^{m \times n}, b \in \mathbb{R}^{m}, a \in \mathbb{R}^{n}$, and $\beta \in \mathbb{R}$ with $P=\mathrm{P} \leq(A, b) \neq \varnothing$. The inequality $\langle a, x\rangle \leq \beta$ is valid for (all $x$ in) $P$ if and only if there is some $\lambda \in \mathbb{R}_{+}^{m}$ with $\lambda^{\mathrm{t}} A=a^{\mathrm{t}}$ and $\langle\lambda, b\rangle \leq \beta$.

Every face $F \neq \varnothing$ of a polyhedron $P=\mathrm{P} \leq(A, b)=\operatorname{conv}(X)+\operatorname{ccone}(Y)$ (with $A \in \mathbb{R}^{m \times n}$, $b \in \mathbb{R}^{m}$, and $X, Y \subseteq \mathbb{R}^{n}$ finite) is a polyhedron as well with

$$
F=\operatorname{conv}(X \cap F)+\operatorname{ccone}(Y \cap \operatorname{char}(F)) .
$$


Moreover, with

$$
I=\mathrm{Eq}_{A x \leq b}(F)=\left\{i \in[m]: F \subseteq \mathrm{H}^{=}\left(A_{i, \star}, b_{i}\right)\right\},
$$

we have $F=\left\{x \in P: A_{I, \star} x=b_{I}\right\}$, and, conversely, for every $I \subseteq[m]$, the set $F=\{x \in P$ : $\left.F \subseteq \mathrm{H}^{=}\left(A_{i, \star}, b_{i}\right)\right\}$ is a face of $P$ with $I \subseteq \operatorname{Eq}_{A x \leq b}(F)$.

In particular, every polyhedron has finitely many faces. Partially ordered by inclusion, they form the face lattice $\mathcal{L}(P)$ of $P$ (including $\varnothing$ and $P$ ). For each face $F \neq \varnothing$ of the polyhedron $P$ we have lineal $(F)=\operatorname{lineal}(P)$. Hence, the (non-empty) faces of pointed polyhedra are pointed polyhedra as well.

The maximal elements in $\mathcal{L}(P) \backslash\{P\}$ are called the facets of $P$. A face of $P$ is a facet if and only if $\operatorname{dim}(F)=\operatorname{dim}(P)-1$ holds (where the dimension $\operatorname{dim}(P)$ of a polyhedron is the affine dimension of its affine hull aff $(P)$ ). The minimal elements in $\mathcal{L}(P) \backslash\{\varnothing\}$ for a pointed polyhedron $P$ are called the vertices of $P$. The vertices of a polyhedron $P$ are the faces of dimension zero, i.e., the faces that contain exactly one point (which, of course, is also called vertex).

Theorem 13. For a point $v \in P$ in a polyhedron $P=\mathrm{P} \leq(A, b)$ (with $A \in \mathbb{R}^{m \times n}$ and $b \in \mathbb{R}^{m}$ ) the following statements are pairwise equivalent.

(1) The point $v$ is a vertex of $P$.

(2) There are no two points $x, x^{\prime} \in P \backslash\{v\}$ with $v \in \operatorname{conv}\left\{x, x^{\prime}\right\}$.

(3) There is some $I \subseteq[m],|I|=n$ such that $A_{I, \star}$ is regular with $v=A_{I, \star}^{-1} b$.

A pointed polyhedral cone $K$ has exactly one vertex, namely $\mathbb{O}$, and therefore, one is more interested in the minimal elements of $\mathcal{L}(K) \backslash\{\varnothing,\{\mathbb{O}\}\}$, which are called the extreme rays of $K$. The extreme rays of a (pointed) polyhedral cone are its faces of dimension one. The one-dimensional unbounded faces of a general pointed polyhedron are called its extreme rays as well, the one dimensional bounded faces are the edges. The edges of a pointed polyhedron $P$ are of the form $\operatorname{conv}\{v, w\}$ with two vertices $v \neq w$ of $P$, and every extreme ray of $P$ can be written as $v+R$ with a vertex $v$ of $P$ and an extreme ray $R$ of $\operatorname{char}(P)$ (which is pointed if $P$ is pointed).

An outer description $P=\left\{x \in \mathbb{R}^{n}: A^{=} x=b^{=}, A^{\leq} x \leq b^{\leq}\right\}$of a polyhedron $P$ is irredundant if removing any equation or any inequalitiy from the system results in a different (larger) polyhedron, and turning any inequality in the system into an equation results in a different (smaller) polyhedron.

Theorem 14. Let $\varnothing \neq P \subseteq \mathbb{R}^{n}$ be a polyhedron, $A^{(1)} \in \mathbb{R}^{m^{(1)} \times n}, b^{(1)} \in \mathbb{R}^{m^{(1)}}, A^{(2)} \in \mathbb{R}^{m^{(2)} \times n}$, and $b^{(2)} \in \mathbb{R}^{m^{(2)}}$ with

$$
P \subseteq\left\{x \in \mathbb{R}^{n}: A^{(1)} x=b^{(1)}, A^{(2)} x \leq b^{(2)}\right\}
$$

such that, for all $i \in\left[m^{(2)}\right]$, we have $P \nsubseteq \mathrm{H}^{=}\left(A^{(2)}{ }_{i, \star}, b_{i}\right)$.

(i) Equality in (5) holds if and only if

- $\operatorname{aff}(P)=\left\{x \in \mathbb{R}^{n}: A^{(1)} x=b^{(1)}\right\}$, and

- for each facet $F$ of $P$, there is an $i \in\left[m^{(2)}\right]$ such that $\left\langle A^{(2)}{ }_{i, \star}, x\right\rangle \leq b_{i}$ defines the facet $F$ of $P$.

(ii) If equality holds in (5), then the right-hand-side of (5) is an irredundant outer description of $P$ if and only if

- $A^{(1)}$ has full row rank, and

- the inequalities in $A^{(2)} x \leq b^{(2)}$ define pairwise distinct facets of $P$.

An inner description $P=\operatorname{conv}(X)+\operatorname{ccone}(Y)$ of a polyhedron $P$ is irredundant if removing any point from $X$ or any vector from $Y$ results in a different (smaller) polyhedron. 
Theorem 15. Let $\varnothing \neq P \subseteq \mathbb{R}^{n}$ be a pointed polyhedron, and let $X, Y \subseteq \mathbb{R}^{n}$ be finite sets with $X \subseteq P$ and $Y \subseteq \operatorname{char}(P)$.

(i) We have $P=\operatorname{conv}(X)+\operatorname{ccone}(Y)$ if and only if

- $X$ contains all vertices of $P$, and

- $Y$ contains a nonzero vector from each extremal ray of $\operatorname{char}(P)$.

(ii) If $P=\operatorname{conv}(X)+\operatorname{ccone}(Y)$ holds, then $\operatorname{conv}(X)+\operatorname{ccone}(Y)$ is an irredundant inner description of $P$ if and only if

- $X$ is the set of vertices of $P$, and

- $Y$ contains exactly one nonzero vector from each extremal ray of $\operatorname{char}(P)$.

As for a pointed polyhedron $P=\operatorname{conv}(X)+\operatorname{ccone}(Y)$ (with finite sets $X, Y \subseteq \mathbb{R}^{n}$ ), $c \in \mathbb{R}^{n}$, and $\omega=\max \{\langle c, x\rangle: x \in P\}$ we have $\omega=\infty$ if $\langle c, y\rangle>0$ for some $y \in Y$, and $\omega=\max \{\langle c, x\rangle$ : $x \in X\}$ otherwise, Theorem 15 implies that a bouned linear optimization problem over a pointed polyhedron $P$ attains its optimum in a vertex of $P$.

1.6. Projections of Polyhedra. We mentioned above that the projection $P=\pi(Q)$ of a polyhedron $Q \subseteq \mathbb{R}^{d}$ via a linear map $\pi: \mathbb{R}^{d} \rightarrow \mathbb{R}^{n}$ with $\pi(y)=T y$ for a matrix $T \in \mathbb{R}^{n \times d}$ is a polyhedron as well, as, for all $X, Y \subseteq \mathbb{R}^{d}$, we have

$$
\pi(\operatorname{conv}(X)+\operatorname{ccone}(Y))=\operatorname{conv}(\pi(X))+\operatorname{ccone}(\pi(Y)) .
$$

We say that a face $F$ of $Q$ is $\pi$-compatible if it can be defined by an inequality $\left\langle T^{\mathrm{t}} a, y\right\rangle \leq \beta$ (valid for $Q$ ) for some $a \in \mathbb{R}^{n}$.

Theorem 16. For a polyhedron $Q \subseteq \mathbb{R}^{d}$, a linear projection $\pi: \mathbb{R}^{d} \rightarrow \mathbb{R}^{n}$, and the polyhedron $P=\pi(Q)$, the map defined via $F \mapsto \pi(F)$ is an isomorphism between the sublattice of $\mathcal{L}(Q)$ formed by the $\pi$-compatible faces and $\mathcal{L}(P)$.

An extended formulation for a polyhedron $P \subseteq \mathbb{R}^{n}$ is an outer description $Q=\mathrm{P}^{\leq}(A, b)$ of some polyhedron $Q \subseteq \mathbb{R}^{d}$ along with a linear projection $\pi: \mathbb{R}^{d} \rightarrow \mathbb{R}^{n}$ with $\pi(Q)=P$. Such an extended formulation of a polyhedron $P$ can be much simpler (e.g., in terms of the number of inequalities) than any outer description of $P$ if the complexity of the facets of $P$ is hidden in lower dimensional parts of the face lattice $\mathcal{L}(Q)$ (see Theorem 16). As, due to

$$
\max \{\langle c, x\rangle: x \in P\}=\max \left\{\left\langle T^{\mathrm{t}} c, y\right\rangle: y \in Q\right\} \quad\left(\text { for all } c \in \mathbb{R}^{n}\right),
$$

linear optimization problems over $P=\pi(Q)$ can be solved by solving linear optimization problems over $Q$, extended formulations play an important role in modern mathematical optimization (see [6]).

We conclude the first part of the article by considering the question how to derive an outer description of a polyhedron from an extended formulation. Here, the fundamental result follows via Theorem 12 .

Theorem 17. Let $Q=\mathrm{P} \leq(D, g) \subseteq \mathbb{R}^{d}$ be a polyhedron with $D \in \mathbb{R}^{q \times d}$ and $g \in \mathbb{R}^{q}$, suppose the linear projection $\pi: \mathbb{R}^{d} \rightarrow \mathbb{R}^{n}$ is defined via $\pi(y)=$ Ty for a matrix $T \in \mathbb{R}^{n \times d}$ and all $y \in \mathbb{R}^{d}$, and let $\bar{T}$ be any matrix whose rows form a basis of $\operatorname{ker}(T)$.

If $L \in \mathbb{R}_{+}^{m \times d}$ is a matrix whose rows generate the projection cone

$$
\left\{\lambda \in \mathbb{R}_{+}^{q}: \lambda^{\mathrm{t}}\left(D \bar{T}^{\mathrm{t}}\right)=\mathbb{O}\right\}=\operatorname{ccone}\left\{L_{1, \star}, \ldots, L_{m, \star}\right\},
$$

then every $A \in \mathbb{R}^{m \times n}$ with $A T=L D$ satisfies

$$
\pi(Q)=\mathrm{P}^{\leq}(A, b) \cap \pi\left(\mathbb{R}^{d}\right)
$$

with $b=L g$. 
If, in the situation of Theorem 17, the projection $\pi: \mathbb{R}^{d} \rightarrow \mathbb{R}^{n}$ is the orthogonal projection to the first $n$ coordinates, then the projection cone is simply

$$
\left\{\lambda \in \mathbb{R}_{+}^{q}:\left\langle D_{\star, j}, \lambda\right\rangle=0 \text { for all } j \in[d] \backslash[n]\right\},
$$

and $A$ can be chosen to consist of the first $n$ columns of $L D$. If furthermore $n=d-1$ holds, then a finite generating system for the projection cone (6) can be obtained from the relation

$$
\left\{\lambda \in \mathbb{R}_{+}^{r+s}: \sum_{i=1}^{r+s} \lambda_{i} \delta_{i}=0\right\}=\operatorname{ccone}\left\{\mathbb{e}_{i}-\mathbb{e}_{k}: i \in[r], k \in[r+s] \backslash[r]\right\}
$$

for all numbers $\delta_{1}, \ldots, \delta_{r}>0>\delta_{r+1}, \ldots, \delta_{r+s}$ (which one can easily establish by induction on $r+$ $s)$.

Theorem 18 (Fourier-Motzkin elimination). For a polyhedron $Q=\mathrm{P} \leq(D, g) \subseteq \mathbb{R}^{d}$ (with $B \in \mathbb{R}^{q \times d}$ and $g \in \mathbb{R}^{q}$ ) and the sets

$$
\begin{aligned}
& I^{>0}=\left\{i \in[q]: D_{i, d}>0\right\} \\
& I^{=0}=\left\{i \in[q]: D_{i, d}=0\right\} \\
& I^{<0}=\left\{i \in[q]: D_{i, d}<0\right\},
\end{aligned}
$$

the polyhedron in $\mathbb{R}^{d-1}$ arising from $Q$ by orthogonal projection to the first $d-1$ coordinates is the set of all $x \in \mathbb{R}^{d-1}$ that satisfy the following system:

$$
\begin{aligned}
\left\langle D_{i, \star}, x\right\rangle & \leq g_{i} & & \text { for all } i \in I^{=0} \\
\left\langle D_{k, d} D_{\ell, \star}-D_{\ell, d} D_{k, \star}, x\right\rangle & \leq D_{k, d} g_{\ell}-D_{\ell, d} g_{k} & & \text { for all } k \in I^{>0}, \ell \in I^{<0}
\end{aligned}
$$

By applying the Fourier-Motzkin elimination method (Theorem 18) iteratedly, one can compute from an outer descriptions of a polyhedron an outer description of its orthogonal projection to any coordinate subspace. Note that the sizes of the descriptions may blow up exponentially (even if, after each iteration, one removes redundant constraints).

The case of general projections can be solved by Fourier-Motzin elimination as follows, where we adopt the notation from above. Let $\tilde{n}$ be the rank of $T$, and assume that the submatrix $T_{[\tilde{n}],[\tilde{n}]}$ of $T$ is regular. Denoting by $\tilde{T} \in \mathbb{R}^{d \times d}$ the regular matrix that equals $T_{\tilde{n}, \star}$ on its first $\tilde{n}$ rows and that has the vectors $\mathbb{e}_{i}$, for $i=\tilde{n}+1, \ldots, d$, in its other rows, and by $\tilde{\pi}: \mathbb{R}^{d} \rightarrow \mathbb{R}^{\tilde{n}}$ the orthogonal projection to the first $\tilde{n}$ coordinates, we have $\pi(y)_{[\tilde{n}]}=\tilde{\pi}(\tilde{T} y)$ for all $y \in \mathbb{R}^{d}$. Thus, with $\tilde{D}=D \tilde{T}^{-1}$ and $\tilde{Q}=\mathrm{P} \leq(\tilde{D}, g)$, we find $P=\left\{x \in \pi\left(\mathbb{R}^{d}\right): x_{\tilde{n}} \in \tilde{\pi}(\tilde{Q})\right\}$.

One reason for the interest in computing projections of polyhedra is that one can convert inner to outer and outer to inner description by means of such computations. Indeed, it follows from the discussion of homogenization and the concept of polarity that all such conversions can be done by any method that computes, for a given finite set $X \subseteq \mathbb{R}^{n}$, a matrix $A \in \mathbb{R}^{m \times n}$ with ccone $(X)=$ $\mathrm{P}^{\leq}(A, \mathbb{O})$ (see Theorem 7). Denoting by $T \in \mathbb{R}^{n \times d}$ a matrix whose set of columns is $X$, we have, by definition, ccone $(X)=\pi(Q)$, where $\pi: \mathbb{R}^{d} \rightarrow \mathbb{R}^{n}$ is the projection defined via $\pi(y)=T y$ for all $y \in \mathbb{R}^{d}$ and $Q=\mathbb{R}_{+}^{d}$. Thus, computing an outer description of $\pi(Q)$ from the outer description $\mathbb{R}_{+}^{d}=\mathrm{P} \leq\left(-\mathrm{Id}_{d}, \mathbb{O}\right)$ of $Q$ solves the problem. 


\section{Polyhedra AND InTEgRALity}

Integral points in polyhedra play a crucial role in integer linear programming and combinatorial optimization. The fundamental concept here is the integer hull

$$
P_{\mathrm{I}}=\operatorname{conv}\left(P \cap \mathbb{Z}^{n}\right)
$$

of a polyhedron $P \subseteq \mathbb{R}^{n}$. In this second part, we describe the most fundamental results on integer hulls, which are important, since, on the one hand, they satisfy

$$
\max \left\{\langle c, x\rangle: x \in P \cap \mathbb{Z}^{n}\right\}=\max \left\{\langle c, x\rangle: x \in P_{\mathrm{I}}\right\}
$$

for all $c \in \mathbb{R}^{n}$, while on the other hand, as convex objects, they are much more convenient to deal with than the discrete sets $P \cap \mathbb{Z}^{n}$. In particular we will treat integral polyhedra, i.e., polyhedra $P$ with $P_{\mathrm{I}}=P$. Identifying a polyhedron $P$ as integral is particularly pleasant, as in this case integer linear optimization problems over $P$ can be treated as (continuous) linear optimization problems over $P$.

Theorem 19. For $A \in \mathbb{Q}^{m \times n}$ and $b \in \mathbb{Q}^{m}$, defining an integral polyhedron $\mathrm{P}^{\leq}(A, b)$, one can, for every $c \in \mathbb{Q}^{n}$, find $x^{\star} \in \mathrm{P}^{\leq}(A, b) \cap \mathbb{Z}^{n}$ with

$$
\left\langle c, x^{\star}\right\rangle=\max \left\{\langle c, x\rangle: x \in \mathrm{P}^{\leq}(A, b) \cap \mathbb{Z}^{n}\right\}
$$

(or conclude that no such $x^{\star}$ exists) in time bounded by a polynomial in $\langle A\rangle+\langle b\rangle+\langle c\rangle$.

2.1. Integer Points in Polyhedra. Similarly to the parameterization (3) of points in a polyhedron by an inner description, for a rational polyhedron $P$, we can also parameterize the set of all integral points in $P$. For a finite set $Y \subseteq \mathbb{R}^{n}$, we denote by

$$
\operatorname{mono}(Y)=\left\{\sum_{y \in Y} \alpha_{y} y: \alpha_{y} \in \mathbb{N} \text { for all } y \in Y\right\}
$$

(with $\mathbb{N}=\{0,1,2, \ldots\}$ ) the submonoid of $\mathbb{R}^{n}$ generated by $Y$.

Theorem 20. For every rational polyhedron $P \subseteq \mathbb{R}^{n}$ there are finite sets $X, Y \subseteq \mathbb{Z}^{n}$ with

$$
P \cap \mathbb{Z}^{n}=X+\operatorname{mono}(Y) \text { and } \operatorname{char}(P)=\operatorname{ccone}(Y),
$$

such that $\langle X \cup Y\rangle_{\max }$ is bounded by a polynomial in $n$ and $\langle P\rangle_{\max }^{\mathrm{inner}}$ (and thus, by a polynomial in $n$ and $\langle P\rangle$ max $)$.

A finite set $H \subseteq \mathbb{Z}^{n}$ is called an integral Hilbert basis (of ccone $(H)$ ) if

$$
\operatorname{ccone}(H) \cap \mathbb{Z}^{n}=\operatorname{mono}(H)
$$

holds. The existence statement of the following theorem is a special case of Theorem 20 .

Theorem 21. Every rational polyhedral cone $K$ has an integral Hilbert basis; if $K$ is pointed, then the integral Hilbert basis of $K$ is uniquely determined.

Moreover, Theorem 20 implies that, whenever the rational system $A x \leq b$ (with $A \in \mathbb{Q}^{m \times n}$ and $b \in \mathbb{Q}^{m}$ ) has any integral solution, then it also has an integral solution whose encoding length is bounded by a polynomial in $\langle(A, b)\rangle$. This shows that the integer linear programming feasibility problem is contained in the complexity class NP (it is, however, not in coNP, unless NP $=$ coNP).

Finally, from Theorem 20 one can derive that the integral hulls of rational polyhedra are rational polyhedra as well.

Theorem 22. For each rational polyhedron $P \subseteq \mathbb{R}^{n}$, the integer hull $P_{\mathrm{I}}$ of $P$ is a rational polyhedron, for which $\left\langle P_{\mathrm{I}}\right\rangle_{\max }^{\text {outer }}$ is bounded by a polynomial in $n$ and $\langle P\rangle_{\max }^{\text {outer }}$. 
The number of facets of $P_{\mathrm{I}}$ is, however, in general not bounded polynomially in the number of facets of $P$. Furthermore, the integer hull of a non-rational polyhedron needs not even be a polyhedron (see, e.g., $\left.P=\left\{\left(x_{1}, x_{2}\right) \in \mathbb{R}^{2}: x_{2} \leq \sqrt{2} x_{1}, x_{1} \geq 1\right\}\right)$.

A generalization of Theorem 22 to mixed-integer hulls of rational polyhedra holds as well: If $P \subseteq \mathbb{R}^{n}$ is a rational polyhedron, then $\operatorname{conv}\left\{x \in P: x_{J} \in \mathbb{Z}^{J}\right\}$ is a rational polyhedron for every $J \subseteq[n]$.

We end this section by a crucial criterion for integrality of (pointed rational) polyhedra.

Theorem 23. A pointed rational polyhedron is integral (i,e, $P_{\mathrm{I}}=P$ ) if and only if all vertices of $P$ are integral.

2.2. Total Dual Integrality. A rational system $A x \leq b$ with $A \in \mathbb{Q}^{m \times n}$ and $b \in \mathbb{Q}^{m}$ defining a non-empty polyhedron $\mathrm{P} \leq(A, b) \neq \varnothing$ is called totally dual integral (TDI) if, for every $c \in \mathbb{Z}^{n}$ with $\max \{\langle c, x\rangle: A x \leq b\}<\infty$, there is some $y^{\star} \in \mathbb{N}^{m}$ with $A^{\mathrm{t}} y^{\star}=c$ and

$$
\left\langle b, y^{\star}\right\rangle=\min \left\{\langle b, y\rangle: A^{\mathrm{t}} y=c, y \in \mathbb{R}_{+}^{m}\right\},
$$

i.e., the dual problem to $\max \{\langle c, x\rangle: A x \leq b\}$ has an integral optimal solution $y^{\star}$. It is convenient to consider TDI all rational systems $A x \leq b$ with $\mathrm{P} \leq(A, b)=\varnothing$ as well.

Theorem 24. A system $A x \leq b$ with integral matrix $A \in \mathbb{Z}^{m \times n}$ and rational right-hand-side vector $b \in \mathbb{Q}^{m}$ is TDI if and only if, for every face $F \neq \varnothing$ of $\mathrm{P}^{\leq}(A, b)$, the set

$$
\left\{A_{i, \star}: i \in \mathrm{Eq}_{A x \leq b}(F)\right\}
$$

is an integral Hilbert basis.

Applying Theorem 21 to the rational normal cones

$$
\left\{c \in \mathbb{R}^{n}:\left\langle c, x^{\star}\right\rangle=\max \{\langle c, x\rangle: x \in P\} \text { for all } x^{\star} \in F\right\}
$$

of the (minimal) faces $F$ of a rational polyhedron $P$, one can construct TDI-systems as in the following result.

Theorem 25. Let $P \subseteq \mathbb{R}^{n}$ be a rational polyhedron.

(1) There is an integral matrix $A \in \mathbb{Z}^{m \times n}$ and a rational vector $b \in \mathbb{Q}^{m}$ with $P=\mathrm{P} \leq(A, b)$ such that $A x \leq b$ is TDI, where $b$ can be chosen to be integral if $P$ is integral.

(2) If there is a rational matrix $A \in \mathbb{Q}^{m \times n}$ and an integral vector $b \in \mathbb{Z}^{m}$ with $P=\mathrm{P} \leq(A, b)$ such that $A x \leq b$ is TDI, then $P$ is integral.

Thus, every rational polyhedron admits an outer descriptions by a TDI-system with integral (lefthand-side) coefficient matrix, though, in general, this will not be an irredundant description in the sense of Section 1.5 Nevertheless, such a description can provide much structural insight, e.g., within the theory of cutting planes for non-integral polyhedra. The algorithmic problem, however, to decide whether a given rational system $A x \leq b$ is TDI is coNP-complete (even for $A$ restricted to the set of node-edge incidence matrices, see Section 2.3, of undirected graphs).

Outer descriptions of (integral) polyhedra with integral right-hand-side vectors are very important, because due to Theorem 25 2) (and the definition of TDI) they yield strong duality relations for certain integer linear optimization problems.

Theorem 26. If the system $A x \leq b$ is TDI with $A \in \mathbb{Q}^{m \times n}$ and an integral right-hand side $b \in \mathbb{Z}^{m}$, and $P=\mathrm{P} \leq(A, b) \neq \varnothing$ holds, then, for every $c \in \mathbb{Z}^{n}$ with $\max \{\langle c, x\rangle: x \in P\}<\infty$, we have

$$
\max \left\{\langle c, x\rangle: A x \leq b, x \in \mathbb{Z}^{n}\right\}=\min \left\{\langle b, y\rangle: A^{\mathrm{t}} y=c, y \geq \mathbb{O}, y \in \mathbb{Z}^{m}\right\} .
$$


2.3. Total Unimodularity. A matrix $A \in\{-1,0,1\}^{m \times n}$ is totally unimodular (TU) if all square submatrices of $A$ have determinant $-1,0$, or 1 . The (version for pointed polyhedra of the) following result is a consequence of Theorem 23 and Theorem 13 (see part (3)) obtained by Cramer's rule (Theorem 2).

Theorem 27. If $A \in\{-1,0,1\}^{m \times n}$ is totally unimodular and $b \in \mathbb{Z}^{m}$ is integral, then $\mathrm{P} \leq(A, b)$ is an integral polyhedron.

If $A \in\{-1,0,1\}^{m \times n}$ is totally unimodular, then so are all submatrices of $A,\left(A, \operatorname{Id}_{m}\right),(A,-A)$, and $A^{\mathrm{t}}$, where the latter allows to conclude the following from Theorem 27

Theorem 28. If $A \in\{-1,0,1\}^{m \times n}$ is totally unimodular, then, for every $b \in \mathbb{Q}^{m}$, the system $A \leq b$ is TDI.

In particular, if $A \in\{-1,0,1\}^{m \times n}$ is totally unimodular, then the strong duality relation (7) holds for all integral $b \in \mathbb{Z}^{m}$ and $c \in \mathbb{Z}^{n}$ (for which the respective optimal values are finite).

The following criterion is extremely useful for establishing total unimodularity of matrices.

Theorem 29 (Criterion of Ghouila-Houri). A matrix $A \in\{-1,0,1\}^{m \times n}$ is totally unimodular if and only if, for every subset $I \subseteq[\mathrm{m}]$ of row indices, there is a partitioning $I=I^{+} \uplus I^{-}$(with $I^{+} \cap I^{-}=\varnothing$ ) such that

$$
\sum_{i \in I^{+}} A_{i, \star}-\sum_{i \in I^{-}} A_{i, \star} \in\{-1,0,1\}^{n}
$$

holds. (Clearly, a similar characterization via all subsets of column indices holds.)

From Theorem 29 one readily deduces that every matrix with entries from $\{-1,0,1\}$ that has at most one positive and at most one negative entry per column is totally unimodular. In particular, the node-arc incidence matrix $\operatorname{inc}(D) \in\{-1,0,1\}^{V \times A}$ of a directed graph $D=(V, A)$ (with $A \subseteq$ $V \times V)$, defined via

$$
\operatorname{inc}(D)_{v, a}= \begin{cases}-1 & \text { if } a=(v, w) \text { for some } w \in V \\ 1 & \text { if } a=(u, v) \text { for some } u \in V \\ 0 & \text { otherwise }\end{cases}
$$

for all $v \in V$ and $a \in A$, is totally unimodular. Thus, the set

$$
\left\{x \in \mathbb{R}^{A}: \operatorname{inc}(D) x=\mathbb{O}, \ell \leq x \leq u\right\}
$$

of circulations in $D$ respecting the integral lower and upper bounds $\ell, u \in \mathbb{Z}^{A}$ is an integral polytope.

The node-edge incidence matrix $\operatorname{inc}(G) \in\{0,1\}^{V \times E}$ of an undirected graph $G=(V, E)$ is definied via

$$
\operatorname{inc}(G)_{v, e}= \begin{cases}1 & \text { if } e=\{v, w\} \text { for some } w \in V \\ 0 & \text { otherwise }\end{cases}
$$

for all $v \in V$ and $e \in E$. Theorem 29 yields that the node-edge incidence matrix of a graph $G=$ $(V, E)$ is totally unimodular if and only if the graph is bipartite, i.e., there is a partitioning $V=S \uplus T$ (with $S \cap T=\varnothing$ ) such that $E \subseteq\{\{s, t\}: s \in S, t \in T\}$. In particular, the matching polytope of a bipartite graph $G=(V, E)$ (i.e., the convex hull of the characteristic vectors $\chi(M) \in\{0,1\}^{E}$-with $\chi(M)_{e}=1$ if and only if $e \in M-$ of all matchings $M \subseteq E$ - with $e \cap e^{\prime}=\varnothing$ for all $e, e^{\prime} \in M$, 
$\left.e \neq e^{\prime}\right)$ has

$$
\begin{aligned}
\sum_{e \in E: v \in e} x_{e} \leq 1 & \text { for all } v \in V \\
x_{e} \geq 0 & \text { for all } e \in E
\end{aligned}
$$

as an outer description.

The most important class of totally unimodular matrices is formed by the network matrices. Such a network matrix $N \in\{-1,0,1\}^{A_{T} \times A}$ arises from a directed graph $D=(V, A)$ and a subset $A_{T} \subseteq A$ of $|V|-1$ arcs that, viewed as undirected edges, form a spanning tree on $V$, by setting, for each $a^{\prime} \in A_{T}$ and $a=(v, w) \in A$,

$$
N_{a^{\prime}, a}= \begin{cases}1 & \text { if the } v \text {-w-path in } A_{T} \text { uses } a^{\prime} \text { in forward direction } \\ -1 & \text { if the } v \text {-w-path in } A_{T} \text { uses } a^{\prime} \text { in backward direction } \\ 0 & \text { otherwise }\end{cases}
$$

Theorem 30. Network matrices are totally unimodular.

In fact, network matrices are the crucial building blocks of the whole class of totally unimodular matrices, as every totally unimodular matrix arises from networks matrices and two special totally unimodular $5 \times 5$-matrices by certain operations that preserve total unimodularity. From such structural results one can derive polynomial time algorithms for testing matrices $A$ for total unimodularity (while, as remarked at the end of Section 2.2, testing a system $A x \leq b$ for total dual integrality is coNP-complete).

\section{Pointers to Literature}

Whenever possible, we provide, for the results mentioned in the article, pointers to proofs in [33], which is also a great source for historical notes and many more references than listed here.

The Farkas-Lemma (Theorem 1) dates back to work by Farkas as well as by Minkowski at the end of the 19th century (see [33, Sect. 7.3]). There are several possibilities to prove the theorem. In fact, the Farkas-Lemma is a special case of more general separation theorems in convex analysis (see, e.g., [31, Chap. 2]). A particularly nice and purely linear algebraic proof is due to Conforti, Di Summa, and Zambelli [8], who derive the Farkas-Lemma from the corresponding obstruction of the solvability of linear equation systems. Theorem 2 is due to Cramer (1750) (see [33, Sect. 3.1]). Theorem 3 follows from [33, Thm. 3.2] (see also [12]). Theorem 4] is due to Carathéodory [5] from 1911 (see also [33, Thm. 7.1]). Theorems [6 7, and 8 have their origins in the work of Farkas, Minkowski [28], and Weyl [39] (see also [33, Sect. 7.2]). The statements on the components of the vectors and on the entries of the matrices (as well as Theorem 9) follow, e.g., from (the proofs in) [33, Sect. 10.2]. An elementary proof of Theorem 6 can be found in [24] (see also [33, Cor. 7.1a]). For proofs of Theorems 10 and 11 (on the characteristic cones and lineality spaces of polyhedra), we refert to [33, Sect. 8.2]. Theorem 12 is Cor. 7.1h in [33]. For the other statements in Section 1.5] see [33, Sect. 8.3-8.9]. Theorem 16] is folklore (we are not aware of any other explicit reference, thus we refer to [25]). Theorem 17] usually is formulated for orthogonal projections to coordinate subspaces only (see, e.g., [7, Sect. 2.4]). An explicit proof in the general setting can be found in [25]. The Fourier-Motzkin method (Theorem 18) is treated in [33, Sect. 12.2]. The method is due to Motzkin [29], where the idea goes back to work of Fourier in the early 19th century. For the algorithmic problem of converting representations of polyhedra, we refer to the survey by Seidel [35] and to the software system polymake by Gawrilow and Joswig [15] (http://www.opt.tu-darmstadt.de/polymake/). 
The proof of Theorem 19 relies on both that (continuous) linear programs (Khachiyan [22]) as well as systems of linear Diophantine equations can be solved in polynomial time (see [33, Thm. 16.2]). For proofs of Theorems 20, 21, and22] we refer to [33, Sect. 16.2-16.4, 17.2]. The fact that the integer hull of a rational polyhedron is a rational polyedron (Theorem 22] is due to Meyer [27], the notion of Hilbert bases has been introduced by Giles and Pulleyblank [17], where the ideas of the proof of Theorem 21 date back to Gordan [18]. The concept of total dual integrality has been invented by Edmonds and Giles [13, 14]. See [33, Sect. 22.3] for proofs of Theorems 24] and 25] (the results being due to [17] and Schrijver [32]). The coNP-hardness of the TDI-property has been established by Ding, Feng, and Zang [11]. Proofs of the results on total unimodularity can be found in [33. Chap. 19]. The connection between totaly unimodular matrices and integral polyhedra (Theorem 27 and a similar characterization of total unimodularity) is due to Hoffman and Kruskal [23]. Theorem 29 has been proved by Ghouila-Houri [16]. The total unimodularity of network matrices (Theorem 30) is due to Tutte [38]. The decomposition theorem for totally unimodular matrices mentioned after Theorem 30] has been proved by Seymour [36]. Cunningham and Edmonds [10] derived a polynomial time test for total unimodularity from that theorem, the asymptotically fastest known algorithm is due to Truemper [37].

\section{REFERENCES}

[1] Alexander Barvinok. A course in convexity, volume 54 of Graduate Studies in Mathematics. American Mathematical Society, Providence, RI, 2002.

[2] Margaret M. Bayer and Carl W. Lee. Combinatorial aspects of convex polytopes. In Handbook of convex geometry, Vol. A, B, pages 485-534. North-Holland, Amsterdam, 1993.

[3] Dimitris Bertsimas and Robert Weismantel. Optimization over Integers. Dynamic Ideas, 2005.

[4] Rainer E. Burkard. Convexity and discrete optimization. In Handbook of convex geometry, Vol. A, B, pages 675-698. North-Holland, Amsterdam, 1993.

[5] Constantin Carathéodory. Gesammelte mathematische Schriften. Bd. 3, chapter Über den Variabilitätsbereich der Fourierschen Konstanten von positiven harmonischen Funktionen, pages 78-110. C. H. Beck'sche Verlagsbuchhandlung, München, 1955. Herausgegeben im Auftrag und mit Unterstützung der Bayerischen Akademie der Wissenschaften.

[6] Michele Conforti, Gerard Cornuéjols, and Giacomo Zambelli. Extended formulations in combinatorial optimization. Technical report, Università di Padova, 2009.

[7] Michele Conforti, Gerard Cornuéjols, and Giacomo Zambelli. 50 Years of Integer Programming 1958-2008, chapter Polyhedral Approaches to Mixed Integer Linear Programming. Springer, 2009 (to appear).

[8] Michele Conforti, Marco Di Summa, and Giacomo Zambelli. Minimally infeasible set-partitioning problems with balanced constraints. Math. Oper. Res., 32(3):497-507, 2007.

[9] William J. Cook, William H. Cunningham, William R. Pulleyblank, and Alexander Schrijver. Combinatorial optimization. Wiley-Interscience Series in Discrete Mathematics and Optimization. John Wiley \& Sons Inc., New York, 1998. A Wiley-Interscience Publication.

[10] William H. Cunningham and Jack Edmonds. A combinatorial decomposition theory. Canad. J. Math., 32(3):734-765, 1980.

[11] Guoli Ding, Li Feng, and Wenan Zang. The complexity of recognizing linear systems with certain integrality properties. Math. Programming, 114(2):321-334, 2008.

[12] Jack Edmonds. Systems of distinct representatives and linear algebra. J. Res. Nat. Bur. Standards Sect. B, 71B:241245, 1967.

[13] Jack Edmonds and Rick Giles. A min-max relation for submodular functions on graphs. In Studies in integer programming (Proc. Workshop, Bonn, 1975), pages 185-204. Ann. of Discrete Math., Vol. 1. North-Holland, Amsterdam, 1977.

[14] Jack Edmonds and Rick Giles. Total dual integrality of linear inequality systems. In Progress in combinatorial optimization (Waterloo, Ont., 1982), pages 117-129. Academic Press, Toronto, ON, 1984.

[15] Ewgenij Gawrilow and Michael Joswig. polymake: a framework for analyzing convex polytopes. In Gil Kalai and Günter M. Ziegler, editors, Polytopes - Combinatorics and Computation, pages 43-74. Birkhäuser, 2000. 
[16] Alain Ghouila-Houri. Caractérisation des matrices totalement unimodulaires. C. R. Acad. Sci. Paris, 254:1192-1194, 1962.

[17] F. R. Giles and W. R. Pulleyblank. Total dual integrality and integer polyhedra. Linear Algebra Appl., 25:191-196, 1979.

[18] P. Gordan. Ueber die Auflösung linearer Gleichungen mit reellen Coefficienten. Math. Ann., 6(1):23-28, 1873.

[19] Peter Gritzmann and Victor Klee. Mathematical programming and convex geometry. In Handbook of convex geometry, Vol. A, B, pages 627-674. North-Holland, Amsterdam, 1993.

[20] Martin Grötschel, László Lovász, and Alexander Schrijver. Geometric algorithms and combinatorial optimization, volume 2 of Algorithms and Combinatorics. Springer-Verlag, Berlin, second edition, 1993.

[21] Branko Grünbaum. Convex polytopes, volume 221 of Graduate Texts in Mathematics. Springer-Verlag, New York, second edition, 2003. Prepared and with a preface by Volker Kaibel, Victor Klee and Günter M. Ziegler.

[22] L. G. Hačijan. A polynomial algorithm in linear programming. Dokl. Akad. Nauk SSSR, 244(5):1093-1096, 1979.

[23] A. J. Hoffman and J. B. Kruskal. Integral boundary points of convex polyhedra. In Linear inequalities and related systems, Annals of Mathematics Studies, no. 38, pages 223-246. Princeton University Press, Princeton, N. J., 1956.

[24] Volker Kaibel. Another proof of the fact that polyhedral cones are finitely generated. http://arxiv.org/abs/0912.2927, 2009.

[25] Volker Kaibel. Two theorems on projections of polyhedra. Available from: http://www.math.uni-magdeburg.de/ kaibel/Downloads/ProjectPoly.pdf Dec 2009.

[26] Bernhard Korte and Jens Vygen. Combinatorial optimization, volume 21 of Algorithms and Combinatorics. SpringerVerlag, Berlin, fourth edition, 2008. Theory and algorithms.

[27] R. R. Meyer. On the existence of optimal solutions to integer and mixed-integer programming problems. Math. Programming, 7:223-235, 1974.

[28] Hermann Minkowski. Geometry of numbers. (Geometrie der Zahlen.). Bibliotheca Mathematica Teubneriana. 40. New York, NY: Johnson Reprint Corp. vii, 256 p. , 1968.

[29] Theodor S. Motzkin. Beiträge zur Theorie der linearen Ungleichungen. PhD thesis, Basel: Diss. 73 S. , 1936.

[30] George Nemhauser and Laurence Wolsey. Integer and combinatorial optimization. Wiley-Interscience Series in Discrete Mathematics and Optimization. John Wiley \& Sons Inc., New York, 1999. Reprint of the 1988 original, A Wiley-Interscience Publication.

[31] Andrzej Ruszczyński. Nonlinear optimization. Princeton University Press, Princeton, NJ, 2006.

[32] A. Schrijver. On total dual integrality. Linear Algebra Appl., 38:27-32, 1981.

[33] Alexander Schrijver. Theory of linear and integer programming. Wiley-Interscience Series in Discrete Mathematics. John Wiley \& Sons Ltd., Chichester, 1986. A Wiley-Interscience Publication.

[34] Alexander Schrijver. Polyhedral combinatorics. In Handbook of combinatorics, Vol. 1, 2, pages 1649-1704. Elsevier, Amsterdam, 1995.

[35] Raimund Seidel. Convex hull computations. In Jacob E. Goodman and Joseph O'Rourke, editors, Handbook of Discrete and Computational Geometry, chapter 24, pages 495-512. CRC Press LLC, Boca Raton, FL, 2004.

[36] P. D. Seymour. Decomposition of regular matroids. J. Combin. Theory Ser. B, 28(3):305-359, 1980.

[37] Klaus Truemper. A decomposition theory for matroids. V. Testing of matrix total unimodularity. J. Combin. Theory Ser. B, 49(2):241-281, 1990.

[38] W. T. Tutte. Lectures on matroids. J. Res. Nat. Bur. Standards Sect. B, 69B:1-47, 1965.

[39] H. Weyl. Elementare Theorie der konvexen Polyeder. Commentarii math. Helvetici, 7:290-306, 1935.

[40] Laurence A. Wolsey. Integer programming. Wiley-Interscience Series in Discrete Mathematics and Optimization. John Wiley \& Sons Inc., New York, 1998. A Wiley-Interscience Publication.

[41] Günter M. Ziegler. Lectures on polytopes, volume 152 of Graduate Texts in Mathematics. Springer-Verlag, New York, 1995.

VOLKER KAIBEL

E-mail address: kaibel@ovgu.de 